\title{
Twisted Breit-Wheeler electron-positron pair creation via vortex gamma photons
}

\author{
Zhigang Bu, ${ }^{1}$ Liangliang Ji $\odot,{ }^{1, *}$ Shaohu Lei $\odot,{ }^{1,2}$ Huayu $\mathrm{Hu},{ }^{3}$ Xiaomei Zhang, ${ }^{4}$ and Baifei Shen ${ }^{4,1, \dagger}$ \\ ${ }^{1}$ State Key Laboratory of High Field Laser Physics and CAS Center for Excellence in Ultra-intense Laser Science, Shanghai Institute of \\ Optics and Fine Mechanics (SIOM), Chinese Academy of Sciences (CAS), Shanghai 201800, China \\ ${ }^{2}$ University of Chinese Academy of Sciences, Beijing 100049, China \\ ${ }^{3}$ Hypervelocity Aerodynamics Institute, China Aerodynamics Research and Development Center, Mianyang, Sichuan 621000, China \\ ${ }^{4}$ Department of Physics, Shanghai Normal University, Shanghai 200234, China
}

(Received 7 September 2020; accepted 11 November 2021; published 7 December 2021)

\begin{abstract}
Particles in quantum vortex states (QVSs) carrying definite orbital angular momenta (OAM) bring different perspectives in various fundamental interaction processes. When unique properties arise in the QVS, understanding how OAM manifests itself between initial particles and the outcome in vortex particle collisions becomes essential. This is made possible by applying the complete vortex description for all involved particles such that angular momenta (AM) are represented by explicit quantum numbers and their connections are naturally retrieved. We demonstrate the full-vortex quantum-electrodynamics (QED) results for the Breit-Wheeler pair creation process and derive the AM-dependent selection rule. The numerically resolved cross sections show antisymmetric spin polarization and, most importantly, the OAM spectra in vortex collision processes. The latter reveals efficient conversion of OAM to created pairs, leading to featured hollow and ring-shaped structure in the density distribution. These results demonstrate a clear picture in understanding the AM physics in the scattering processes of high energy particles.
\end{abstract}

DOI: 10.1103/PhysRevResearch.3.043159

\section{INTRODUCTION}

The experimental advances in production and measurement of electrons carrying orbital angular momentum (OAM) [1-4] have attracted extensive interest in this decade. In addition to spin angular momentum (SAM), electron beams containing OAM are closely associated with the vortex structure, also known as the "twisted" modes. Theoretical description of the relativistic vortex electrons is based on the spinor Bessel mode $[5,6]$ as an exact solution of the free Dirac equation. In analog to photon [7] and scalar particle states [8], the quantum vortex state (QVS) in Bessel mode can be interpreted as the superposition of the plane wave states according to a particular phase structure. Alternatively, direct comparison with the optical vortex field $[9,10]$ leads to another vortex state, the Laguerre-Gauss mode [11,12], under the paraxial approximation. These vortex states have shown several unique features, including spin-orbital coupling [5,13], spin-orbital conversion [14], spin and orbital Hall effects [15,16], and geometric phase [17]. The OAM degree of freedom manifests itself in various interaction processes such as the vortex electron state dressed in laser field

\footnotetext{
*jill@siom.ac.cn

†bfshen@mail.shcnc.ac.cn
}

Published by the American Physical Society under the terms of the Creative Commons Attribution 4.0 International license. Further distribution of this work must maintain attribution to the author(s) and the published article's title, journal citation, and DOI.
[18,19], Compton scattering [7], elastic electron-atom scattering [20,21], two-photon annihilation of vortex positron [22], resonance production in vortex particle collisions [23], radiation from vortex electrons [24,25], and strong-field ionization [26-28]. The ongoing studies have brought up new perspectives in high energy physics, nuclear physics, atomic dynamics, strong-field physics, and in producing exotic vortex light sources.

In a broad range of particle collisions, transferring of polarization and OAM from the initial states to the final ones is of central interest. Governed by the conservation law of total angular momentum (TAM), this important connection can be revealed from the angular momentum (AM) dependent cross sections. The latter depends on resolving the complete vortex scattering in which all interacting particles are described by the QVS, as a plane wave does not carry OAM. Up to now, however, due to the complexity of the QVS, the quantum electrodynamics (QED) picture of complete vortex scattering remains to be seen.

In this article, we apply the first full-vortex description on the Breit-Wheeler (BW) pair creation process, where OAM properties appear in both photon fields and created fermion particles. To describe the annihilation of two QVS gamma photons into a QVS electron-positron pair, we employ the vortex states for both and derive the scattering cross section within the theoretical framework of QED. This allows AM to be explicitly expressed by the quantum numbers and inherently conserved. The AM-dependent selection rules and the OAM spectra are illustrated. Together they show how OAM is transferred from the initial gamma photons to final electrons and positrons, which also depends on the asymmetric 
polarization distribution. Consequently, the generated pairs exhibit periodic ring-shape and hollow structure in the density distribution, providing a unique feature for identifying the vortex scattering process.

\section{DEFINITION OF QUANTUM VORTEX STATES: BESSEL MODES}

The particles carrying definite OAM can be described by the QVS (also known as the "twisted state"). The QVS is defined by the Bessel modes which are con- structed from the superposition of plane wave (PW) states via $\Phi_{k_{\perp}, k_{z}}^{s, l}(x)=\int d^{3} \boldsymbol{k}^{\prime} \tilde{\varphi}_{k_{\perp}, k_{z}}^{l}\left(\boldsymbol{k}^{\prime}\right) \Phi_{\boldsymbol{k}^{\prime}}^{s}(x)$ in cylindrical momentum space $\boldsymbol{k}^{\prime}=\left(k_{\perp}^{\prime}, \phi_{k}^{\prime}, k_{z}^{\prime}\right)$ [7], assuming the particle propagates along the $z$ axis. Here $\Phi_{\boldsymbol{k}^{\prime}}^{s}(x)$ denotes a PW scalar, vector, or spinor particle state, and $\tilde{\varphi}_{k_{\perp}, k_{z}}^{l}\left(\boldsymbol{k}^{\prime}\right)=$ $1 /\left(\sqrt{2 \pi} i^{l} k_{\perp}\right) \delta\left(k_{z}^{\prime}-k_{z}\right) \delta\left(k_{\perp}^{\prime}-k_{\perp}\right) e^{i l \phi^{\prime}{ }_{k}}$ is the Fourier spectrum containing a spiral phase with OAM number $l$ and $s$ represents the spin if the particle carries. Under this definition, the QVS of the photon field takes the form where $\lambda= \pm 1$ is the polarization parameter, $\omega$ is the photon energy, $j$ is the total angular momentum (TAM) of the QVS photon, and the transverse function is defined by $\Theta_{k_{\perp}}^{n}(\boldsymbol{r})=$ $J_{n}\left(k_{\perp} r\right) e^{i n \theta}\left[J_{n}(r)\right.$ is the Bessel function of the first kind]. The photon QVS satisfies the orthonormality: $\left(A_{k_{\perp}, k_{z}}^{j, \lambda}, A_{\boldsymbol{k}_{\perp}^{\prime}, \boldsymbol{k}_{z}^{\prime}}^{j^{\prime}, \lambda^{\prime}}\right)=$ $-\left(1 / k_{\perp}\right) \delta_{\lambda \lambda^{\prime}} \delta_{j j^{\prime}} \delta\left(k_{\perp}-k_{\perp}^{\prime}\right) \delta\left(k_{z}-k_{z}^{\prime}\right)$. We use the natural units $\hbar=c=1$ throughout the derivation. Analogously, the QVS of electron and positron can be constructed from the positive and negative-frequency PW solutions of the Dirac equation, and they are given by

$$
\psi_{p_{\perp}, p_{z}}^{ \pm, l, s}(x)=\frac{e^{ \pm i p_{z} z \mp i E t}}{\sqrt{2}(2 \pi)|\boldsymbol{p}|} \sqrt{1-\frac{M}{E}}\left[\chi_{z}^{ \pm} \Theta_{p_{\perp}}^{l}(\boldsymbol{r}) \pm i p_{\perp} \chi_{\perp}^{ \pm}\right]
$$

where $M$ and $E$ are electron/positron mass and energy. $\chi_{z}^{ \pm}$ and $\chi_{\perp}^{ \pm}$represent the spinor wave functions in longitudinal and transverse dimensions. For an electron, $\chi_{z}^{+}=\left(\begin{array}{c}(E+M) \xi^{s} \\ p_{z} \sigma_{z} \xi^{s}\end{array}\right)$ and $\chi_{\perp}^{+}=\left(\begin{array}{c}0 \\ \sigma_{\perp}^{l, p} p_{\perp}\end{array}\right)$; for a positron, $\chi_{z}^{-}=\left(\begin{array}{c}p_{z} \sigma_{z} \eta^{s} \\ (E+M) \eta^{s}\end{array}\right)$ and $\chi_{\perp}^{-}=$ $\left(\begin{array}{c}\sigma_{\perp}^{l, p_{\perp}} \eta^{s} \\ 0\end{array}\right)$, with transversal matrix $\sigma_{\perp}^{l, p_{\perp}}=\left(\begin{array}{cc}0 & -\Theta_{p_{\perp}}^{l-1}(\boldsymbol{r}) \\ \Theta_{p_{\perp}}^{l+1}(\boldsymbol{r}) & 0\end{array}\right)$. $\xi^{s}$ and $\eta^{s}$ are two basis spinors characterizing the electron and positron spin states in the rest frame. The spinor QVS satisfies the orthonormality $\left(\psi_{p_{\perp}, p_{z}}^{ \pm, l, s}, \psi_{p_{\perp}^{\prime}, p_{z}^{\prime}}^{ \pm, s^{\prime}}\right)=$ $\left(1 / p_{\perp}\right) \delta_{s s^{\prime}} \delta_{l l^{\prime}} \delta\left(p_{\perp}-p^{\prime}{ }_{\perp}\right) \delta\left(p_{z}-p_{z}^{\prime}\right)$. One finds that due to the spin-orbit interaction of relativistic particles, the QVS is neither the eigenmodes of the projection of the OAM operator $\hat{L}_{z}$ nor the SAM operator $\hat{S}_{z}$ on the $z$ axis, but rather of the TAM operator $\hat{J}_{z}=\hat{L}_{z}+\hat{S}_{z}$. For a vortex photon, the eigenvalue of $\hat{J}_{z}$ is $j: \hat{J}_{z} A_{k_{\perp}, k_{z}, \mu}^{j, \lambda}=j A_{k_{\perp}, k_{z}, \mu}^{j, \lambda}$; the vortex electron/positron gives $\hat{J}_{z} \psi_{p_{\perp}, p_{z}}^{ \pm, l, s}=(l+s / 2) \psi_{p_{\perp}, p_{z}}^{ \pm, l, s}$.

\section{S-MATRIX AND SCATTERING CROSS SECTION OF VORTEX BW PROCESS}

In perturbation theory, the $S$ matrix for the vortex BW process contains two terms: $S_{f i}=S_{1}+S_{2}$. The Feynman diagram for $S_{1}$ is drawn in Fig. 1(a), where the cone on each external line represents a QVS and its polar angle is defined by $\tan \alpha_{p}=p_{\perp} / p_{z}$. This diagram is calculated as

$$
S_{1}=-i e^{2} \int d^{4} x d^{4} x^{\prime} \frac{d^{4} q}{(2 \pi)^{4}} \bar{\psi}_{p_{1 \perp}, p_{1 z}}^{+, l_{1}, s_{1}}(x) \mathcal{A}_{k_{1 \perp}, k_{1 z}}^{j_{1}, \lambda_{1}}(x) \frac{(\not 1+M)}{\left(q^{2}-M^{2}\right)} e^{-i q \cdot\left(x-x^{\prime}\right)} \mathcal{A}_{k_{2 \perp}, k_{2 z}}^{j_{2}, \lambda_{2}}\left(x^{\prime}\right) \psi_{p_{2 \perp}, p_{2 z}}^{-, l_{2}, s_{2}}\left(x^{\prime}\right)
$$

with the slash $\not A=A_{\mu} \gamma^{\mu}$. We consider the forward and backward scatterings here. Substituting the eigenmodes of photon field (1) and electron/positron field (2) into Eq. (3), one obtains

$$
\begin{aligned}
& S_{1}=-\frac{i e^{2}}{16 \pi} \sqrt{\frac{\left(E_{1}-M\right)\left(E_{2}-M\right)}{\omega_{1} \omega_{2} E_{1} E_{2} \boldsymbol{p}_{1}^{2} \boldsymbol{p}_{2}^{2}}} \delta\left(\omega_{1}+\omega_{2}-E_{1}-E_{2}\right) \delta\left(k_{1 z}+k_{2 z}-p_{1 z}-p_{2 z}\right) \\
& \times\left.\xi^{s_{1} \dagger} \Xi_{k_{1 \perp}, k_{1 z} ; k_{2 \perp}, k_{2 z}}^{j_{1}, \lambda_{1} ; j_{2}, \lambda_{2}}\left(l_{1}, p_{1 \perp}, p_{1 z} ; l_{2}, p_{2 \perp}, p_{2 z}\right) \eta^{s_{2}}\right|_{\substack{E_{q}=-\omega_{1}+E_{1}=\omega_{2}-E_{2} \\
q_{z}=-k_{1 z}+p_{1 z}=k_{2 z}-p_{2 z}}},
\end{aligned}
$$

where the $(2 \times 2)$ matrix

$$
\Xi_{k_{1 \perp}, k_{1 z} ; k_{2 \perp}, k_{2 z}}^{j_{1}, \lambda_{1} ; j_{2}, \lambda_{2}}\left(l_{1}, p_{1 \perp}, p_{1 z} ; l_{2}, p_{2 \perp}, p_{2 z}\right)=\left(\begin{array}{cc}
v_{11} \delta_{j_{1}+j_{2}, l_{1}-l_{2}} & v_{12} \delta_{j_{1}+j_{2}, l_{1}-l_{2}+1} \\
v_{21} \delta_{j_{1}+j_{2}, l_{1}-l_{2}-1} & v_{22} \delta_{j_{1}+j_{2}, l_{1}-l_{2}}
\end{array}\right) .
$$



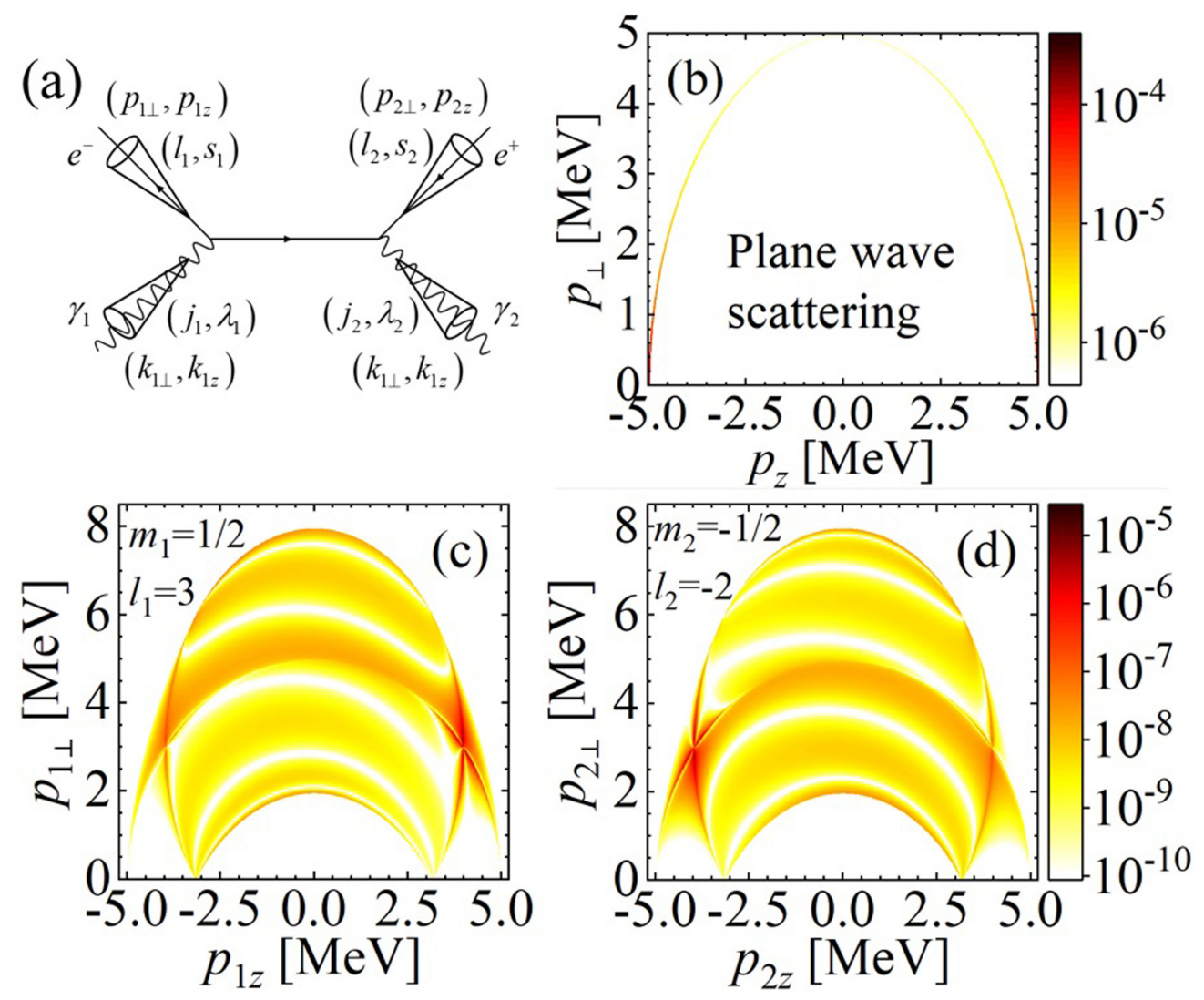

FIG. 1. (a) Feynman diagram of matrix element $S_{1}$; the cones represent the QVS particle. (b) Differential cross sections of electrons, $d \sigma\left(e^{-}\right) / d p_{\perp} d p_{z}\left[\mathrm{MeV}^{-4}\right]$, in cylindrical momentum space created in BW process with plane-wave particle states. The energies of head-on colliding photons are $\omega_{1}=\omega_{2}=5 \mathrm{MeV}$. Differential cross sections of electrons $d \sigma\left(e^{-}\right) / d p_{1 \perp} d p_{1 z}\left[\mathrm{MeV}^{-4}\right]$ (c) and positrons $d \sigma\left(e^{+}\right) / d p_{2 \perp} d p_{2 z}\left[\mathrm{MeV}^{-4}\right](\mathrm{d})$ in vortex BW process. The energies and $z$ momenta of the QVS photons are $\omega_{1}=\omega_{2}=5 \mathrm{MeV}$ and $k_{1 z}=-k_{2 z}=4 \mathrm{MeV}$, the AM parameters are $j_{1}=3, j_{2}=2, \lambda_{1}=\lambda_{2}=1$. The AM of created electron and positron are $l_{1}=3, m_{1}=1 / 2$ and $l_{2}=-2, m_{2}=-1 / 2$.

The matrix elements $v_{11}, v_{12}, v_{21}$, and $v_{22}$ are derived in the Appendix. The second term $S_{2}$ can be obtained by exchanging two incident photons in $S_{1}, \Xi$ matrix and matrix elements $v: S_{2}, \tilde{\Xi}, \tilde{v}=S_{1}, \Xi, v\left(k_{1} \leftrightarrow k_{2}, j_{1} \leftrightarrow j_{2}, \lambda_{1} \leftrightarrow \lambda_{2}\right)$. It is found that each matrix element in $\Xi$ and $\tilde{\Xi}$ has an AMdependent Kronecker delta function, which gives the selection rules for the vortex BW process,

$$
j_{1}+j_{2}=l_{1}-l_{2}+\Delta,
$$

with $\Delta=0, \pm 1$. The minus sign before $l_{2}$ stems from the definition of positron AM. Using the $S$-matrix element one obtains the spin-dependent pair creation probability: $d \mathcal{P}=$ $\left(2 \pi^{2} /(R L)\right)^{2} k_{1 \perp} k_{2 \perp} p_{1 \perp} p_{2 \perp}\left|S_{f i}\right|^{2} d p_{1 \perp} d p_{1 z} d p_{2 \perp} d p_{2 z}$, where $R$ and $L$ are the large radius and length used in the cylindrical normalization. The differential cross section is derived as $d \sigma=d \mathcal{P} /\left(T\left\langle u_{z}\right\rangle\right)$. For a head-on collision, the relative current density of the incident photons is given by $\left\langle u_{z}\right\rangle=$ $\left|k_{1 z} / \omega_{1}-k_{2 z} / \omega_{2}\right| / V$, and the cross section reads

$$
\begin{aligned}
d \sigma= & \frac{\pi^{3} \alpha^{2}}{16} \frac{k_{1 \perp} k_{2 \perp} p_{1 \perp} p_{2 \perp}\left(E_{1}-M\right)\left(E_{2}-M\right)}{\omega_{1} \omega_{2} E_{1} E_{2} \boldsymbol{p}_{1}^{2} \boldsymbol{p}_{2}^{2}\left|k_{1 z} / \omega_{1}-k_{2 z} / \omega_{2}\right|} \operatorname{Tr}\left[\xi^{s_{1}} \xi^{s_{1} \dagger}(\Xi+\tilde{\Xi}) \eta^{s_{2}} \eta^{s_{2} \dagger}(\Xi+\tilde{\Xi})^{\dagger}\right] \\
& \times \delta\left(\omega_{1}+\omega_{2}-E_{1}-E_{2}\right) \delta\left(k_{1 z}+k_{2 z}-p_{1 z}-p_{2 z}\right) d p_{1 \perp} d p_{1 z} d p_{2 \perp} d p_{2 z} .
\end{aligned}
$$

For the unpolarized photon scattering, the cross section can be obtained by averaging over the photon polarization $\lambda_{1}$ and $\lambda_{2}$.

The selection rules from Eq. (6) are related to the spin states of electron and position in the rest frame. If $\xi^{s_{1}}$ is the eigenmode of $\sigma_{z}$ for an electron with the eigenvalue $s_{1}=+1, \quad \xi^{s_{1}}=\left(\begin{array}{l}1 \\ 0\end{array}\right)$, and $\eta^{s_{2}}$ for positron with $s_{2}=+1, \eta^{s_{2}}=\left(\begin{array}{l}1 \\ 0\end{array}\right)$, the trace in Eq. (7) is given by $\operatorname{Tr}\left[\xi^{s_{1}} \xi^{s_{1} \dagger}(\Xi+\tilde{\Xi}) \eta^{s_{2}} \eta^{s_{2} \dagger}(\Xi+\tilde{\Xi})^{\dagger}\right]=$ $\left|v_{11}+\tilde{v}_{11}\right|^{2} \delta_{j_{1}+j_{2}, l_{1}-l_{2}}$. Since the positron has the reversed $\mathrm{AM}$ definition with respect to the electron, i.e., the eigenvalue of the state $\eta^{s_{2}}=\left(\begin{array}{l}1 \\ 0\end{array}\right)$ is $m_{2}=-1 / 2$, the TAM in the $z$ axis of the created electron-positron pair is $l_{1}-l_{2}$, corresponding to 

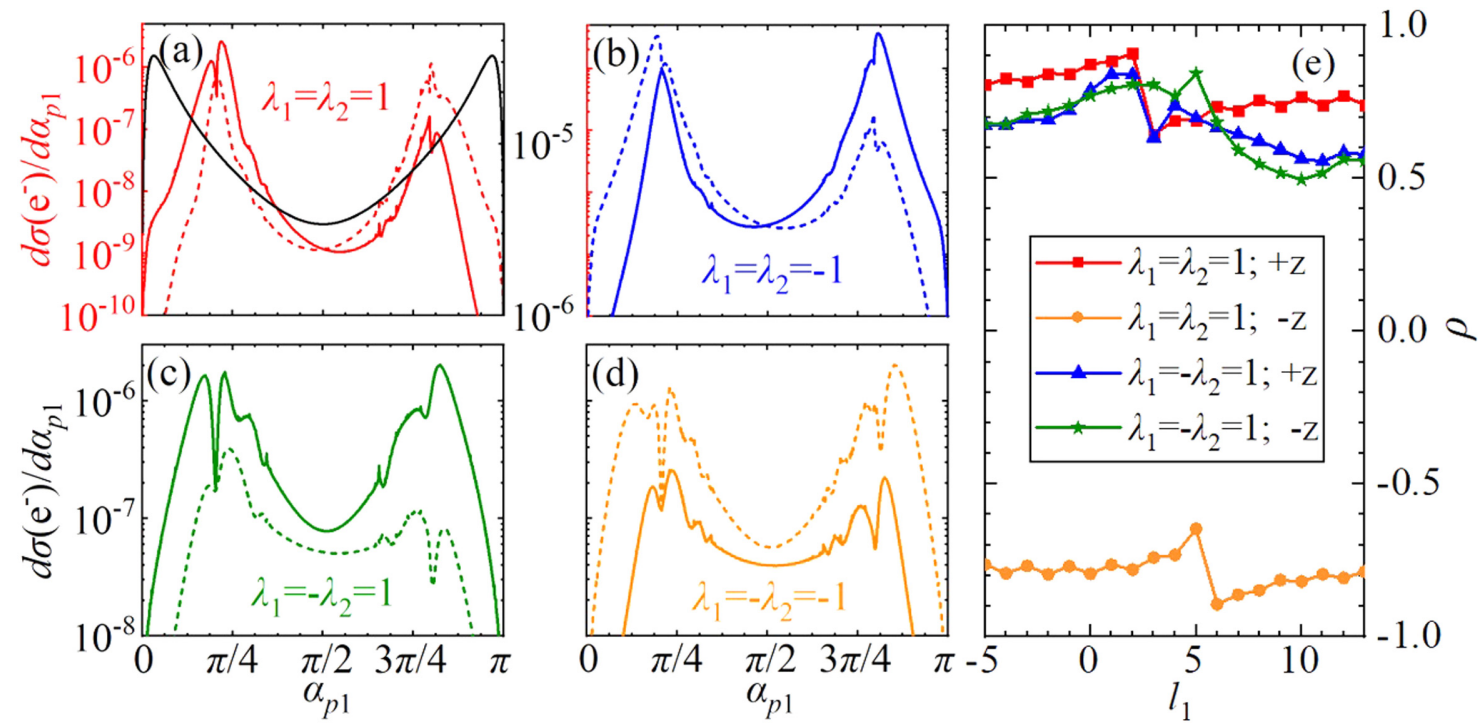

FIG. 2. (a)-(d): $\alpha_{p 1}$-dependent cross sections $d \sigma\left(e^{-}\right) / d \alpha_{p 1}\left[\mathrm{MeV}^{-2}\right]$ of electrons carrying the AM of $l_{1}=3$ for different photon polarizations. The TAMs of the gamma photons are $j_{1}=3$ and $j_{2}=5$, energies $\omega_{1}=\omega_{2}=5 \mathrm{MeV}$ and $z$ momenta $k_{1 z}=-k_{2 z}=4 \mathrm{MeV}$. The colored solid lines represent the angular distributions of electrons with spin $m_{1}=1 / 2$ in the rest frame, the dashed lines represent electrons with spin $m_{1}=-1 / 2$. The black solid line in Fig. 2(a) represents the electron angular distribution created in the BW process with plane-wave particle states. (e) Polarizability of electrons scattered into $+z$ and $-z$ directions versus the electron OAM number $l_{1}$.

the conservation law: $j_{1}+j_{2}=l_{1}-l_{2}$, which agrees with the selection rule of $\Delta=0$. Similarly, $\Delta=+1,-1$ correspond to $m_{1}=m_{2}=+1 / 2$ and $m_{1}=m_{2}=-1 / 2$, respectively. The $m_{1}=-m_{2}=-1 / 2$ case is consistent with the selection rule of $\Delta=0$. In other words, Eq. (6) concludes TAM conservation in the $z$ direction in the vortex BW process. The cross section of non-spin-polarized pairs is obtained by summing over the pair spin states.

\section{NUMERICAL RESULTS}

The cross section for electrons/positrons can be numerically obtained by integrating over the positron/electron momentum in Eq. (7). This is done trivially by dropping the two delta functions. We choose an example of two vortex photons with energies $\omega_{1}=\omega_{2}=5 \mathrm{MeV}$ and $z$ momenta $k_{1 z}=$ $-k_{2 z}=4 \mathrm{MeV}$. The TAMs of two QVS photons are $j_{1}=3$ and $j_{2}=2$, with polarization $\lambda_{1}=\lambda_{2}=1$, respectively. Figures 1(c) and 1(d) show the differential cross sections of electron and positron, where the AMs are $l_{1}=3, m_{1}=1 / 2$ (electron) and $l_{2}=-2, m_{2}=-1 / 2$ (positron) following the selection rule $j_{1}+j_{2}=l_{1}-l_{2}$. We find the cross sections exhibit oscillating distributions in the momentum domain. The structures of electron and positron are symmetrically correlated, governed by the conservation law of energy and momentum. We thus focus on the electron spectrum in the following.

In general, the $\mathrm{BW}$ process with $\mathrm{PW}$ particle states leads to monoenergetic spectra due to the constraint of four-dimensional momentum conservation. This is shown in Fig. 1(b) as a comparison. The electron cross sections in a head-on collision of two PW photons $\left(\omega_{1}=\omega_{2}=5 \mathrm{MeV}\right)$ exhibit a half circle with an exact $5-\mathrm{MeV}$ radius. This is significantly different from the vortex situation [Figs. 1(c) and 1(d)], since the QVS particle has transverse momentum perpendic- ular to its properation direction. The transverse momentum is characterized by the magnitude, while the azimuthal angle is integrated when constructing the QVS [5,7].

From Fig. 1 one notices that if the QVS gamma photons are polarized, e.g., $\lambda_{1}=\lambda_{2}=1$, the spectral distributions with definite spin projections are asymmetrical with respect to $\pm\left|p_{z}\right|$, which implies spin polarization for the created particles. The differential cross section $d \sigma\left(e^{-}\right) / d \alpha_{p_{1}}$ is displayed in Fig. 2. Here we follow the photon energy and momentum employed in Fig. 1 but vary the photon polarization $\left(\lambda_{1}\right.$ and $\left.\lambda_{2}\right)$ and electron/positron spin state $\left(m_{1}\right.$ and $\left.m_{2}\right)$. First of all, we see that unlike the PW BW process which has the highest cross section near the axis [black-solid line in Fig. 2(a)], the one for the vortex BW process peaks off axis, due to the transverse momentum in the QVS. The electron with $l_{1}=3$ and $m_{1}=+1 / 2$ [red-solid line in Fig. 2(a)] comes from two positron AM channels: $l_{2}=-5, m_{2}=-1 / 2$ and $l_{2}=-4, m_{2}=+1 / 2$. The channels are switched to $l_{2}=-6$, $m_{2}=-1 / 2$ and $l_{2}=-5, m_{2}=+1 / 2$ when the spin state is flipped to $m_{1}=-1 / 2$ [red-dashed line]. However, these channels are not equally distributed. The former is almost one order of magnitude higher than the latter in the region $\alpha_{p_{1}}<\pi / 2$ and lower in $\alpha_{p_{1}}>\pi / 2$. As a result, electrons emitted into $\alpha_{p_{1}}<\pi / 2$ are characterized with a significant positive polarization $\left(m_{1}=+1 / 2\right)$ and a negative one in the opposite direction. This trend also applies for $\lambda_{1}=\lambda_{2}=-1$, except that the polarization distributions is reversed, shown in Fig. 2(b).

When photons have opposite polarizations $\lambda_{1}=-\lambda_{2}=$ 1 , the cross section with $m_{1}=+1 / 2$ is much higher than that with $-1 / 2$ in the full angular space $\alpha_{p_{1}} \in$ $[0, \pi]$, giving $+1 / 2$ spin-polarized electrons in all directions as shown in Fig. 2(c). Naturally, $\lambda_{1}=-\lambda_{2}=$ -1 creates $-1 / 2$ spin-polarization [Fig. 2(d)]. The electron polarizability along the $\pm z$ directions is quantified 

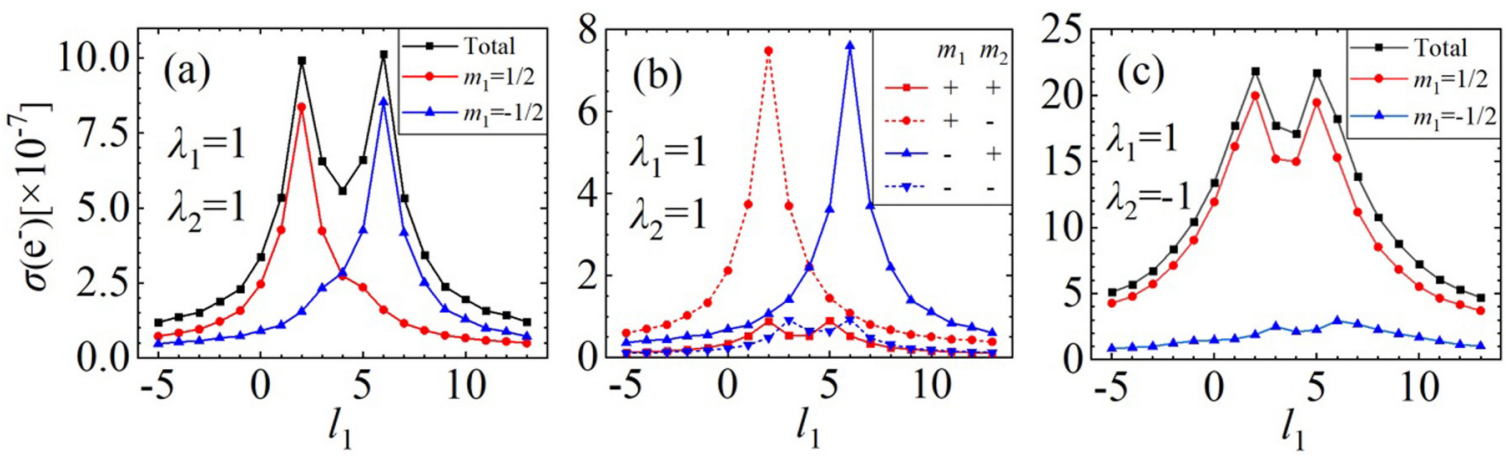

FIG. 3. Electron cross sections $\sigma\left(e^{-}\right)\left[\mathrm{MeV}^{-2}\right]$ versus electron OAM $l_{1}$. The incident gamma photons are polarized, and possess the TAMs $j_{1}=3$ and $j_{2}=5$, energies $\omega_{1}=\omega_{2}=5 \mathrm{MeV}$ and $z$ momenta $k_{1 z}=-k_{2 z}=4 \mathrm{MeV}$. The photon polarization parameters are $\lambda_{1}=\lambda_{2}=1$ (a) and $\lambda_{1}=-\lambda_{2}=1$ (c). (b) Electron cross sections $\sigma\left(e^{-}\right)\left[\mathrm{MeV}^{-2}\right]$ for $\lambda_{1}=\lambda_{2}=1$ polarized photons from different electron/positron polarization channels.

via $\rho=\left[\sigma_{+1 / 2}\left(e^{-}\right)-\sigma_{-1 / 2}\left(e^{-}\right)\right] /\left[\sigma_{+1 / 2}\left(e^{-}\right)+\sigma_{-1 / 2}\left(e^{-}\right)\right]$in Fig. 2(e). One sees that $|\rho|$ is above 0.5 at all OAM numbers $l_{1}$, approaching 0.9 in a certain region. Note that for initially unpolarized photons the created electron-positron pair carrying different spins has almost identical distribution, i.e., polarization vanishes.

A key interest of vortex interaction processes is how it is related to the particle OAM numbers. We show the OAMdependent cross section in Fig. 3. The total cross section peaks at $l_{1}=2$ and 6 , which correspond to the spins $m_{1}=1 / 2$ and $-1 / 2$, as shown in Fig. 3(a). The peaking values are shifted by one unit with respect to the photon TAM $\left(j_{1}=3\right.$, $j_{2}=5$ ). In fact, the OAM peak at $l_{1}=2$ is mainly converted from a photon with $j_{1}=3$ through the dominating channel $m_{1}=1 / 2$ and $m_{2}=-1 / 2$; see the red lines in Fig. 3(b). The other channel of $m_{2}=1 / 2$ is significantly suppressed from the polarization dependence discussed in Fig. 2(a). The blue lines indicate that the peak at $l_{1}=6$ originates from the photon TAM $j_{2}=5$, while the polarization channel $m_{1}=-1 / 2$ and $m_{2}=-1 / 2$ dominates. This connection can also be seen when varying the photon polarization. For instance, with $\lambda_{1}=$ $-\lambda_{2}=1$, since the $m_{1}=1 / 2$ spin state dominates the electron creation over $m_{1}=-1 / 2$, see Fig. 2(c), electrons with an OAM number peaking at $l_{1}=3$ and 6 are suppressed, leading to the main cross section at $l_{1}=2$ and 5, seen in Fig. 3(c). In other words, the OAM-dependent cross section explicitly reveals the OAM number of photons in certain polarization state. The one-unit shift induced by the latter would be negligible at large AM numbers $|j| \gg 1$.

The electron QVS manifests itself in the transverse density distribution. From Eq. (2) we obtain the AM-dependent probability density

$$
\begin{aligned}
\rho_{\perp ; p_{1 \perp}, p_{1 z}}^{+; l_{1}, s_{1}}= & \frac{1}{2(2 \pi)^{2}}\left\{\left[\left(1+\frac{M}{E_{1}}\right)+\left(1-\frac{M}{E_{1}}\right) \frac{p_{1 z}^{2}}{\left|\boldsymbol{p}_{1}\right|^{2}}\right] J_{l_{1}}^{2}\left(p_{1 \perp} r\right)\right. \\
& \left.+\frac{p_{1 \perp}^{2}}{\left|\boldsymbol{p}_{1}\right|^{2}}\left(1-\frac{M}{E_{1}}\right) J_{l_{1}+s_{1}}^{2}\left(p_{1 \perp} r\right)\right\} .
\end{aligned}
$$

This is shown in Fig. 4(a) of the eigenstate with electron energy $E_{1}=5 \mathrm{MeV}$, transverse momentum $p_{1 \perp}=3 \mathrm{MeV}, l_{1}=$ 3 , and $m_{1}=1 / 2$. A periodic ring-shaped profile is seen with a hollow on the axis. The peak density on each ring declines at larger radii. We determine the radius $R_{0}$ of the maximum density via $d \rho_{\perp ; p_{1 \perp}, p_{1 z}}^{+; l_{1}, s_{1}} / d r=0$ (the first ring) and relate it to the polar angle in Fig. 4(b). These dependencies suggest a clear connection between the OAM and the radius of the ring structure with known spin states.

In principle, the ring-shaped structure for monoenergetic electrons in Fig. 4(a) could be retrieved by extracting electrons within a small energy spread, implying a potential scheme to identify the AM of high-energy polarized gamma photon. In reality, the created pairs in the vortex BW process are not monoenergetic. When considering the momentum spectrum distribution generated in a scattering process as the weighting factor, the transverse density of the created electrons can be obtained by summing each monoenergetic electron density. From Fig. 4(c) we notice that the transverse density oscillation is significantly weakened or even offset in this case. However, the hollow density structure is always present, as a unique feature of the vortex modes and hence can be seen as a robust signature to identify the vortex scattering process.

\section{CONCLUSIONS}

In conclusion, we have examined the vortex BW pair creation process based on the QED theory. The full-vortex cross sections clearly reveal the connections of AM between initial photons and final pairs, showing dependence on polarization and OAM numbers. The vortex scattering process leads to distinctive hollow and ring-shaped density distribution of the created pairs.

On the experimental side, the generation of twisted electrons and photons have been realized at low energies by using the spiral phase plate or fork grating [1,2,9,29]. However, these methods do not work for gamma photons since their wavelength is orders of magnitude smaller than the optical wave. An attractive scheme to generate the QVS gamma photons is based on the linear Compton scattering [7], where the low-energy twisted optical photons are scattered off from energetic electrons and blueshifted to gamma photons. To improve the production efficiency, a promising method could be the nonlinear Compton scattering with strong lasers of relativistic intensities. The OAMs of generated gamma photons can originate from the OAM of strong vortex laser (Bessel or Laguerre-Gaussian mode) [30], and it is achievable using off-axis spiral phase mirrors [31]; alternatively, they can 

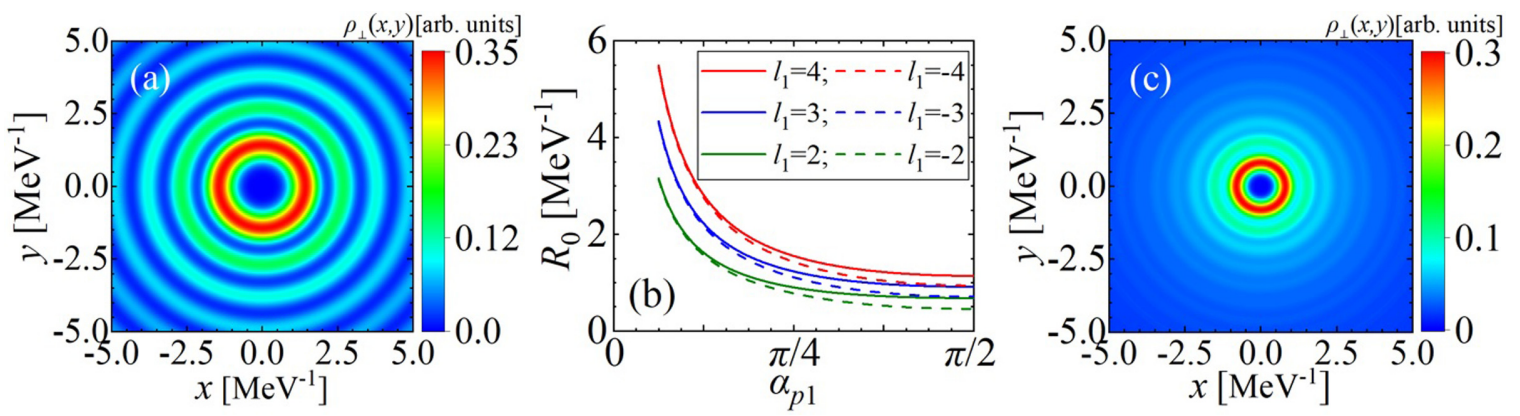

FIG. 4. (a) Transverse density distribution of a monoenergetic QVS electron with energy $E_{1}=5 \mathrm{MeV}$, transverse momentum $p_{1 \perp}=$ $3 \mathrm{MeV}$, AMs $l_{1}=3$ and $m_{1}=1 / 2$. (b) Radius $R_{0}$ of first maximum density versus the polar angle of electron momentum for electron spin $s_{1}=1$ and different OAM $l_{1}$. (c) Transverse density of the created electrons $\left(l_{1}=3\right.$ and $\left.m_{1}=1 / 2\right)$ in a vortex BW process.

also be transferred from the spin polarizations of circularly polarized laser photons.

\section{ACKNOWLEDGMENTS}

This work was supported by the Ministry of Science and Technology of the People's Republic of China (Grants No. 2018YFA0404803 and No. 2016YFA0401102), the National Natural Science Foundation of China (Grants No. 11875307, No. 11935008, and No. 11774415), and the Strategic Priority
Research Program of Chinese Academy of Sciences (Grant No. XDB16010000).

\section{APPENDIX: DERIVATION OF $S$-MATRIX ELEMENTS}

The $S_{1}$ matrix in Eq. (3) contains a transverse phase $e^{i q_{\perp} \cdot \boldsymbol{r}}$ which can be expanded into the AM modes $e^{i n \theta}$ in cylindrical coordinates,

$$
e^{i \boldsymbol{q}_{\perp} \cdot \boldsymbol{r}}=\sum_{n} i^{n} J_{n}\left(q_{\perp} r\right) e^{i n \theta-i n \phi_{q}} .
$$

Substituting Eqs. (1), (2), and (A1) into Eq. (3), one obtains

$$
\begin{aligned}
& S_{1}=-\frac{i e^{2}}{8(2 \pi)^{3}} \sqrt{\frac{\left(E_{1}-M\right)\left(E_{2}-M\right)}{\omega_{1} \omega_{2} E_{1} E_{2} p_{1}^{2} p_{2}^{2}}} \delta\left(\omega_{1}+\omega_{2}-E_{1}-E_{2}\right) \delta\left(k_{1 z}+k_{2 z}-p_{1 z}-p_{2 z}\right) \\
& \times \sum_{n, n^{\prime}} i^{n-n^{\prime}} \int \frac{d q_{\perp} q_{\perp}}{\left(q^{2}-M^{2}\right)}\left[\left(p_{1 z} \xi^{s_{1} \dagger} \sigma_{z} \Gamma_{k_{1 \perp}, k_{1 z}}^{j_{1}, \lambda_{1}},\left(E_{1}+M\right) \xi^{s_{1} \dagger} \Gamma_{k_{1 \perp}, k_{1 z}}^{j_{1}, \lambda_{1}}\right)\right. \\
& \left.-i p_{1 \perp}\left(\xi^{s_{1} \dagger} \tilde{\Gamma}_{k_{1 \perp}, k_{1 z}}^{j_{1}, \lambda_{1}}, 0\right)\right]\left(\begin{array}{cc}
\left(E_{q}+M\right) \delta_{n n^{\prime}} & -Q_{q \perp, q_{z}}^{n, n^{\prime}} \\
Q_{q \perp, q_{z}}^{n, n^{\prime}} & -\left(E_{q}-M\right) \delta_{n n^{\prime}}
\end{array}\right) \\
& \times\left.\left[\left(\begin{array}{c}
\left(E_{2}+M\right) \Gamma_{k_{2 \perp}, k_{2 z}}^{\prime j_{2}, \lambda_{2}} \eta^{s_{2}} \\
-p_{2 z} \Gamma_{k_{2 \perp}, k_{2 z}}^{\prime j_{2}, \lambda_{2}} \sigma_{z} \eta^{s_{2}}
\end{array}\right)+i p_{2 \perp}\left(\begin{array}{c}
0 \\
\tilde{\Gamma}_{k_{2 \perp}, k_{2 z}}^{\prime j_{2}, \lambda_{2}} \eta^{s_{2}}
\end{array}\right)\right]\right|_{\begin{array}{l}
\sum_{q}=-\omega_{1}+E_{1}=\omega_{2}-E_{2} \\
q_{z}=-k_{1 z}+p_{1 z}=k_{2 z}-p_{2 z}
\end{array}},
\end{aligned}
$$

in which there are five $(2 \times 2)$ matrixes:

$$
\begin{gathered}
\Gamma_{k_{1 \perp}, k_{1 z}}^{j_{1}, \lambda_{1}}=\int d \theta d r r J_{n}\left(q_{\perp} r\right) J_{l_{1}}\left(p_{1 \perp} r\right) e^{i\left(n-l_{1}\right) \theta} \Lambda_{k_{1 \perp}, k_{1 z}}^{j_{1}, \lambda_{1}}(r, \theta), \\
\tilde{\Gamma}_{k_{1 \perp}, k_{1 z}}^{j_{1}, \lambda_{1}}=\int d \theta d r r J_{n}\left(q_{\perp} r\right) e^{\mathrm{i} n \theta} \sigma_{\perp}^{l_{1}, p_{1 \perp} \dagger}(r, \theta) \Lambda_{k_{1 \perp}, k_{1 z}}^{j_{1}, \lambda_{1}}(r, \theta), \\
\Gamma_{k_{2 \perp}, k_{2 z}}^{j_{2}, \lambda_{2}}=\int d \theta^{\prime} d r^{\prime} r^{\prime} J_{n^{\prime}}\left(q_{\perp} r^{\prime}\right) J_{l_{2}}\left(p_{2 \perp} r^{\prime}\right) e^{i\left(l_{2}-n^{\prime}\right) \theta^{\prime}} \Lambda_{k_{2 \perp}, k_{2 z}}^{j_{2}, \lambda_{2}}\left(r^{\prime}, \theta^{\prime}\right), \\
\tilde{\Gamma}_{k_{2 \perp}, k_{2 z}}^{\prime j_{2}, \lambda_{2}}=\int d \theta^{\prime} d r^{\prime} r^{\prime} J_{n^{\prime}}\left(q_{\perp} r^{\prime}\right) e^{-i n^{\prime} \theta^{\prime}} \Lambda_{k_{2 \perp}, k_{2 z}}^{j_{2}, \lambda_{2}}\left(r^{\prime}, \theta^{\prime}\right) \sigma_{\perp}^{l_{2}, p_{2 \perp}}\left(r^{\prime}, \theta^{\prime}\right), \\
Q_{q_{\perp}, q_{z}}^{n, n^{\prime}}=\frac{1}{2 \pi} \int d \phi_{q} e^{-i\left(n-n^{\prime}\right) \phi_{q}}(\boldsymbol{\sigma} \cdot \boldsymbol{q})=\left(\begin{array}{cc}
q_{z} \delta_{n n^{\prime}} & q_{\perp} \delta_{n^{\prime}, n+1} \\
q_{\perp} \delta_{n^{\prime}, n-1} & -q_{z} \delta_{n n^{\prime}}
\end{array}\right),
\end{gathered}
$$

with

$$
\Lambda_{k_{\perp}, k_{z}}^{j, \lambda}(r, \theta)=\left(\begin{array}{cc}
\frac{\lambda k_{\perp}}{\omega} J_{j}\left(k_{\perp} r\right) e^{i j \theta} & i\left(1+\frac{\lambda k_{z}}{\omega}\right) J_{j-1}\left(k_{\perp} r\right) e^{i(j-1) \theta} \\
i\left(1-\frac{\lambda k_{z}}{\omega}\right) J_{j+1}\left(k_{\perp} r\right) e^{i(j+1) \theta} & -\frac{\lambda k_{\perp}}{\omega} J_{j}\left(k_{\perp} r\right) e^{i j \theta}
\end{array}\right) .
$$


Substituting these equations into (A2), the $S_{1}$ matrix leads to

$$
\begin{aligned}
S_{1}= & -\frac{i e^{2}}{16 \pi} \sqrt{\frac{\left(E_{1}-M\right)\left(E_{2}-M\right)}{\omega_{1} \omega_{2} E_{1} E_{2} \boldsymbol{p}_{1}^{2} \boldsymbol{p}_{2}^{2}}} \delta\left(\omega_{1}+\omega_{2}-E_{1}-E_{2}\right) \delta\left(k_{1 z}+k_{2 z}-p_{1 z}-p_{2 z}\right) \\
& \times\left.\xi^{s_{1} \dagger}\left(\begin{array}{cc}
v_{11} \delta_{j_{1}+j_{2}, l_{1}-l_{2}} & v_{12} \delta_{j_{1}+j_{2}, l_{1}-l_{2}+1} \\
v_{21} \delta_{j_{1}+j_{2}, l_{1}-l_{2}-1} & v_{22} \delta_{j_{1}+j_{2}, l_{1}-l_{2}}
\end{array}\right) \eta^{s_{2}}\right|_{\substack{E_{q}=-\omega_{1}+E_{1}=\omega_{2}-E_{2} \\
q_{z}=-k_{1 z}+p_{1 z}=k_{2 z}-p_{2 z}}},
\end{aligned}
$$

which is consistent with Eq. (4). The four matrix elements in Eq. (A9) are derived as

$$
\begin{aligned}
& v_{11}=\int \frac{d q_{\perp} q_{\perp}}{\left(q^{2}-M^{2}\right)}\left\{\left[p_{1 z} p_{2 z} q_{z}+p_{1 z}\left(E_{2}+M\right)\left(E_{q}+M\right)+p_{2 z}\left(E_{1}+M\right)\left(E_{q}-M\right)\right.\right. \\
& \left.+q_{z}\left(E_{1}+M\right)\left(E_{2}+M\right)\right] \frac{\lambda_{1} k_{1 \perp} \lambda_{2} k_{2 \perp}}{\omega_{1} \omega_{2}} S_{j_{1}}^{l_{1}} \tilde{S}_{j_{2}}^{j_{2}+l_{2}}+\left[p_{1 z} p_{2 z} q_{z}-p_{1 z}\left(E_{2}+M\right)\left(E_{q}+M\right)\right. \\
& \left.-p_{2 z}\left(E_{1}+M\right)\left(E_{q}-M\right)+q_{z}\left(E_{1}+M\right)\left(E_{2}+M\right)\right]\left(1+\frac{\lambda_{1} k_{1 z}}{\omega_{1}}\right)\left(1-\frac{\lambda_{2} k_{2 z}}{\omega_{2}}\right) S_{j_{1}-1}^{l_{1}} \tilde{S}_{j_{2}+1}^{j_{2}+l_{2}+1} \\
& +\left[p_{1 z} p_{2 \perp} q_{z}+p_{2 \perp}\left(E_{1}+M\right)\left(E_{q}-M\right)\right] \frac{\lambda_{1} k_{1 \perp}}{\omega_{1}}\left(1+\frac{\lambda_{2} k_{2 z}}{\omega_{2}}\right) S_{j_{1}}^{l_{1}} \tilde{S}_{j_{2}-1}^{j_{2}+l_{2}} \\
& +\left[p_{1 \perp} p_{2 z} q_{z}-p_{1 \perp}\left(E_{2}+M\right)\left(E_{q}+M\right)\right] \frac{\lambda_{1} k_{1 \perp}}{\omega_{1}}\left(1-\frac{\lambda_{2} k_{2 z}}{\omega_{2}}\right) S_{j_{1}}^{l_{1}+1} \tilde{S}_{j_{2}+1}^{j_{2}+l_{2}+1} \\
& +\left[p_{1 z} p_{2 z} q_{\perp}+q_{\perp}\left(E_{1}+M\right)\left(E_{2}+M\right)\right] \frac{\lambda_{1} k_{1 \perp}}{\omega_{1}}\left(1-\frac{\lambda_{2} k_{2 z}}{\omega_{2}}\right) S_{j_{1}}^{l_{1}} \tilde{S}_{j_{2}+1}^{j_{2}+l_{2}+1} \\
& -\left[p_{1 z} p_{2 z} q_{\perp}+q_{\perp}\left(E_{1}+M\right)\left(E_{2}+M\right)\right] \frac{\lambda_{2} k_{2 \perp}}{\omega_{2}}\left(1+\frac{\lambda_{1} k_{1 z}}{\omega_{1}}\right) S_{j_{1}-1}^{l_{1}} \tilde{S}_{j_{2}}^{j_{2}+l_{2}} \\
& +\left[p_{1 z} p_{2 \perp} q_{z}-p_{2 \perp}\left(E_{1}+M\right)\left(E_{q}-M\right)\right] \frac{\lambda_{2} k_{2 \perp}}{\omega_{2}}\left(1+\frac{\lambda_{1} k_{1 z}}{\omega_{1}}\right) S_{j_{1}-1}^{l_{1}} \tilde{S}_{j_{2}}^{j_{2}+l_{2}+1} \\
& +\left[p_{1 \perp} p_{2 z} q_{z}+p_{1 \perp}\left(E_{2}+M\right)\left(E_{q}+M\right)\right] \frac{\lambda_{2} k_{2 \perp}}{\omega_{2}}\left(1-\frac{\lambda_{1} k_{1 z}}{\omega_{1}}\right) S_{j_{1}+1}^{l_{1}+1} \tilde{S}_{j_{2}}^{j_{2}+l_{2}} \\
& +p_{1 z} p_{2 \perp} q_{\perp} \frac{\lambda_{1} k_{1 \perp} \lambda_{2} k_{2 \perp}}{\omega_{1} \omega_{2}} S_{j_{1}}^{l_{1}} \tilde{S}_{j_{2}}^{j_{2}+l_{2}+1}+p_{1 \perp} p_{2 \perp} q_{z} \frac{\lambda_{1} k_{1 \perp} \lambda_{2} k_{2 \perp}}{\omega_{1} \omega_{2}} S_{j_{1}}^{l_{1}+1} \tilde{S}_{j_{2}}^{j_{2}+l_{2}+1} \\
& -p_{1 \perp} p_{2 z} q_{\perp} \frac{\lambda_{1} k_{1 \perp} \lambda_{2} k_{2 \perp}}{\omega_{1} \omega_{2}} S_{j_{1}}^{l_{1}+1} \tilde{S}_{j_{2}}^{j_{2}+l_{2}}-p_{1 \perp} p_{2 \perp} q_{\perp} \frac{\lambda_{1} k_{1 \perp}}{\omega_{1}}\left(1+\frac{\lambda_{2} k_{2 z}}{\omega_{2}}\right) S_{j_{1}}^{l_{1}+1} \tilde{S}_{j_{2}-1}^{j_{2}+l_{2}} \\
& +p_{1 \perp} p_{2 \perp} q_{\perp} \frac{\lambda_{2} k_{2 \perp}}{\omega_{2}}\left(1-\frac{\lambda_{1} k_{1 z}}{\omega_{1}}\right) S_{j_{1}+1}^{l_{1}+1} \tilde{S}_{j_{2}}^{j_{2}+l_{2}+1}-p_{1 z} p_{2 \perp} q_{\perp}\left(1+\frac{\lambda_{1} k_{1 z}}{\omega_{1}}\right)\left(1+\frac{\lambda_{2} k_{2 z}}{\omega_{2}}\right) S_{j_{1}-1}^{l_{1}} \tilde{S}_{j_{2}-1}^{j_{2}+l_{2}} \\
& +p_{1 \perp} p_{2 \perp} q_{z}\left(1-\frac{\lambda_{1} k_{1 z}}{\omega_{1}}\right)\left(1+\frac{\lambda_{2} k_{2 z}}{\omega_{2}}\right) S_{j_{1}+1}^{l_{1}+1} \tilde{S}_{j_{2}-1}^{j_{2}+l_{2}} \\
& \left.+p_{1 \perp} p_{2 z} q_{\perp}\left(1-\frac{\lambda_{1} k_{1 z}}{\omega_{1}}\right)\left(1-\frac{\lambda_{2} k_{2 z}}{\omega_{2}}\right) S_{j_{1}+1}^{l_{1}+1} \tilde{S}_{j_{2}+1}^{j_{2}+l_{2}+1}\right\} \\
& v_{12}=-i \int \frac{d q_{\perp} q_{\perp}}{\left(q^{2}-M^{2}\right)}\left\{\left[p_{1 z} p_{2 z} q_{z}-p_{1 z}\left(E_{2}+M\right)\left(E_{q}+M\right)+p_{2 z}\left(E_{1}+M\right)\left(E_{q}-M\right)\right.\right. \\
& \left.-q_{z}\left(E_{1}+M\right)\left(E_{2}+M\right)\right] \frac{\lambda_{1} k_{1 \perp}}{\omega_{1}}\left(1+\frac{\lambda_{2} k_{2 z}}{\omega_{2}}\right) S_{j_{1}}^{l_{1}} \tilde{S}_{j_{2}-1}^{j_{2}+l_{2}-1} \\
& +\left[p_{1 z} p_{2 z} q_{z}+p_{1 z}\left(E_{2}+M\right)\left(E_{q}+M\right)-p_{2 z}\left(E_{1}+M\right)\left(E_{q}-M\right)\right. \\
& \left.-q_{z}\left(E_{1}+M\right)\left(E_{2}+M\right)\right] \frac{\lambda_{2} k_{2 \perp}}{\omega_{2}}\left(1+\frac{\lambda_{1} k_{1 z}}{\omega_{1}}\right) S_{j_{1}-1}^{l_{1}} \tilde{S}_{j_{2}}^{j_{2}+l_{2}} \\
& +\left[p_{1 z} p_{2 z} q_{\perp}-q_{\perp}\left(E_{1}+M\right)\left(E_{2}+M\right)\right] \frac{\lambda_{1} k_{1 \perp} \lambda_{2} k_{2 \perp}}{\omega_{1} \omega_{2}} S_{j_{1}}^{l_{1}} \tilde{S}_{j_{2}}^{j_{2}+l_{2}} \\
& -\left[p_{1 z} p_{2 \perp} q_{z}+p_{2 \perp}\left(E_{1}+M\right)\left(E_{q}-M\right)\right] \frac{\lambda_{1} k_{1 \perp} \lambda_{2} k_{2 \perp}}{\omega_{1} \omega_{2}} S_{j_{1}}^{l_{1}} \tilde{S}_{j_{2}}^{j_{2}+l_{2}-1}
\end{aligned}
$$




$$
\begin{aligned}
& +\left[p_{1 \perp} p_{2 z} q_{z}+p_{1 \perp}\left(E_{2}+M\right)\left(E_{q}+M\right)\right] \frac{\lambda_{1} k_{1 \perp} \lambda_{2} k_{2 \perp}}{\omega_{1} \omega_{2}} S_{j_{1}}^{l_{1}+1} \tilde{S}_{j_{2}}^{j_{2}+l_{2}} \\
& -\left[p_{1 z} p_{2 z} q_{\perp}-q_{\perp}\left(E_{1}+M\right)\left(E_{2}+M\right)\right]\left(1+\frac{\lambda_{1} k_{1 z}}{\omega_{1}}\right)\left(1+\frac{\lambda_{2} k_{2 z}}{\omega_{2}}\right) S_{j_{1}-1}^{l_{1}} \tilde{S}_{j_{2}-1}^{j_{2}+l_{2}-1} \\
& -\left[p_{1 z} p_{2 \perp} q_{z}-p_{2 \perp}\left(E_{1}+M\right)\left(E_{q}-M\right)\right]\left(1+\frac{\lambda_{1} k_{1 z}}{\omega_{1}}\right)\left(1-\frac{\lambda_{2} k_{2 z}}{\omega_{2}}\right) S_{j_{1}-1}^{l_{1}} \tilde{S}_{j_{2}+1}^{j_{2}+l_{2}} \\
& +\left[p_{1 \perp} p_{2 z} q_{z}-p_{1 \perp}\left(E_{2}+M\right)\left(E_{q}+M\right)\right]\left(1-\frac{\lambda_{1} k_{1 z}}{\omega_{1}}\right)\left(1+\frac{\lambda_{2} k_{2 z}}{\omega_{2}}\right) S_{j_{1}+1}^{l_{1}+1} \tilde{S}_{j_{2}-1}^{j_{2}+l_{2}-1} \\
& -p_{1 \perp} p_{2 z} q_{\perp} \frac{\lambda_{1} k_{1 \perp}}{\omega_{1}}\left(1+\frac{\lambda_{2} k_{2 z}}{\omega_{2}}\right) S_{j_{1}}^{l_{1}+1} \tilde{S}_{j_{2}-1}^{j_{2}+l_{2}-1}-p_{1 z} p_{2 \perp} q_{\perp} \frac{\lambda_{1} k_{1 \perp}}{\omega_{1}}\left(1-\frac{\lambda_{2} k_{2 z}}{\omega_{2}}\right) S_{j_{1}}^{l_{1}} \tilde{S}_{j_{2}+1}^{j_{2}+l_{2}} \\
& +p_{1 z} p_{2 \perp} q_{\perp} \frac{\lambda_{2} k_{2 \perp}}{\omega_{2}}\left(1+\frac{\lambda_{1} k_{1 z}}{\omega_{1}}\right) S_{j_{1}-1}^{l_{1}} \tilde{S}_{j_{2}}^{j_{2}+l_{2}-1}+p_{1 \perp} p_{2 z} q_{\perp} \frac{\lambda_{2} k_{2 \perp}}{\omega_{2}}\left(1-\frac{\lambda_{1} k_{1 z}}{\omega_{1}}\right) S_{j_{1}+1}^{l_{1}+1} \tilde{S}_{j_{2}}^{j_{2}+l_{2}} \\
& +p_{1 \perp} p_{2 \perp} q_{\perp} \frac{\lambda_{1} k_{1 \perp} \lambda_{2} k_{2 \perp}}{\omega_{1} \omega_{2}} S_{j_{1}}^{l_{1}+1} \tilde{S}_{j_{2}}^{j_{2}+l_{2}-1}-p_{1 \perp} p_{2 \perp} q_{z} \frac{\lambda_{1} k_{1 \perp}}{\omega_{1}}\left(1-\frac{\lambda_{2} k_{2 z}}{\omega_{2}}\right) S_{j_{1}}^{l_{1}+1} \tilde{S}_{j_{2}+1}^{j_{2}+l_{2}} \\
& \left.-p_{1 \perp} p_{2 \perp} q_{z} \frac{\lambda_{2} k_{2 \perp}}{\omega_{2}}\left(1-\frac{\lambda_{1} k_{1 z}}{\omega_{1}}\right) S_{j_{1}+1}^{l_{1}+1} \tilde{S}_{j_{2}}^{j_{2}+l_{2}-1}-p_{1 \perp} p_{2 \perp} q_{\perp}\left(1-\frac{\lambda_{1} k_{1 z}}{\omega_{1}}\right)\left(1-\frac{\lambda_{2} k_{2 z}}{\omega_{2}}\right) S_{j_{1}+1}^{l_{1}+1} \tilde{S}_{j_{2}+1}^{j_{2}+l_{2}}\right\}, \\
& v_{21}=-i \int \frac{d q_{\perp} q_{\perp}}{\left(q^{2}-M^{2}\right)}\left\{\left[p_{1 z} p_{2 z} q_{z}-p_{1 z}\left(E_{2}+M\right)\left(E_{q}+M\right)+p_{2 z}\left(E_{1}+M\right)\left(E_{q}-M\right)\right.\right. \\
& \left.-q_{z}\left(E_{1}+M\right)\left(E_{2}+M\right)\right] \frac{\lambda_{1} k_{1 \perp}}{\omega_{1}}\left(1-\frac{\lambda_{2} k_{2 z}}{\omega_{2}}\right) S_{j_{1}}^{l_{1}} \tilde{S}_{j_{2}+1}^{j_{2}+l_{2}+1} \\
& +\left[p_{1 z} p_{2 z} q_{z}+p_{1 z}\left(E_{2}+M\right)\left(E_{q}+M\right)-p_{2 z}\left(E_{1}+M\right)\left(E_{q}-M\right)\right. \\
& \left.-q_{z}\left(E_{1}+M\right)\left(E_{2}+M\right)\right] \frac{\lambda_{2} k_{2 \perp}}{\omega_{2}}\left(1-\frac{\lambda_{1} k_{1 z}}{\omega_{1}}\right) S_{j_{1}+1}^{l_{1}} \tilde{S}_{j_{2}}^{j_{2}+l_{2}} \\
& -\left[p_{1 z} p_{2 z} q_{\perp}-q_{\perp}\left(E_{1}+M\right)\left(E_{2}+M\right)\right] \frac{\lambda_{1} k_{1 \perp} \lambda_{2} k_{2 \perp}}{\omega_{1} \omega_{2}} S_{j_{1}}^{l_{1}} \tilde{S}_{j_{2}}^{j_{2}+l_{2}} \\
& +\left[p_{1 z} p_{2 \perp} q_{z}+p_{2 \perp}\left(E_{1}+M\right)\left(E_{q}-M\right)\right] \frac{\lambda_{1} k_{1 \perp} \lambda_{2} k_{2 \perp}}{\omega_{1} \omega_{2}} S_{j_{1}}^{l_{1}} \tilde{S}_{j_{2}}^{j_{2}+l_{2}+1} \\
& -\left[p_{1 \perp} p_{2 z} q_{z}+p_{1 \perp}\left(E_{2}+M\right)\left(E_{q}+M\right)\right] \frac{\lambda_{1} k_{1 \perp} \lambda_{2} k_{2 \perp}}{\omega_{1} \omega_{2}} S_{j_{1}}^{l_{1}-1} \tilde{S}_{j_{2}}^{j_{2}+l_{2}} \\
& -\left[p_{1 \perp} p_{2 z} q_{z}-p_{1 \perp}\left(E_{2}+M\right)\left(E_{q}+M\right)\right]\left(1+\frac{\lambda_{1} k_{1 z}}{\omega_{1}}\right)\left(1-\frac{\lambda_{2} k_{2 z}}{\omega_{2}}\right) S_{j_{1}-1}^{l_{1}-1} \tilde{S}_{j_{2}+1}^{j_{2}+l_{2}+1} \\
& +\left[p_{1 z} p_{2 \perp} q_{z}-p_{2 \perp}\left(E_{1}+M\right)\left(E_{q}-M\right)\right]\left(1-\frac{\lambda_{1} k_{1 z}}{\omega_{1}}\right)\left(1+\frac{\lambda_{2} k_{2 z}}{\omega_{2}}\right) S_{j_{1}+1}^{l_{1}} \tilde{S}_{j_{2}-1}^{j_{2}+l_{2}} \\
& +\left[p_{1 z} p_{2 z} q_{\perp}-q_{\perp}\left(E_{1}+M\right)\left(E_{2}+M\right)\right]\left(1-\frac{\lambda_{1} k_{1 z}}{\omega_{1}}\right)\left(1-\frac{\lambda_{2} k_{2 z}}{\omega_{2}}\right) S_{j_{1}+1}^{l_{1}} \tilde{S}_{j_{2}+1}^{j_{2}+l_{2}+1} \\
& -p_{1 z} p_{2 \perp} q_{\perp} \frac{\lambda_{1} k_{1 \perp}}{\omega_{1}}\left(1+\frac{\lambda_{2} k_{2 z}}{\omega_{2}}\right) S_{j_{1}}^{l_{1}} \tilde{S}_{j_{2}-1}^{j_{2}+l_{2}}-p_{1 \perp} p_{2 z} q_{\perp} \frac{\lambda_{1} k_{1 \perp}}{\omega_{1}}\left(1-\frac{\lambda_{2} k_{2 z}}{\omega_{2}}\right) S_{j_{1}}^{l_{1}-1} \tilde{S}_{j_{2}+1}^{j_{2}+l_{2}+1} \\
& +p_{1 \perp} p_{2 z} q_{\perp} \frac{\lambda_{2} k_{2 \perp}}{\omega_{2}}\left(1+\frac{\lambda_{1} k_{1 z}}{\omega_{1}}\right) S_{j_{1}-1}^{l_{1}-1} \tilde{S}_{j_{2}}^{j_{2}+l_{2}}+p_{1 z} p_{2 \perp} q_{\perp} \frac{\lambda_{2} k_{2 \perp}}{\omega_{2}}\left(1-\frac{\lambda_{1} k_{1 z}}{\omega_{1}}\right) S_{j_{1}+1}^{l_{1}} \tilde{S}_{j_{2}}^{j_{2}+l_{2}+1} \\
& -p_{1 \perp} p_{2 \perp} q_{\perp} \frac{\lambda_{1} k_{1 \perp} \lambda_{2} k_{2 \perp}}{\omega_{1} \omega_{2}} S_{j_{1}}^{l_{1}-1} \tilde{S}_{j_{2}}^{j_{2}+l_{2}+1}-p_{1 \perp} p_{2 \perp} q_{z} \frac{\lambda_{1} k_{1 \perp}}{\omega_{1}}\left(1+\frac{\lambda_{2} k_{2 z}}{\omega_{2}}\right) S_{j_{1}}^{l_{1}-1} \tilde{S}_{j_{2}-1}^{j_{2}+l_{2}} \\
& \left.-p_{1 \perp} p_{2 \perp} q_{z} \frac{\lambda_{2} k_{2 \perp}}{\omega_{2}}\left(1+\frac{\lambda_{1} k_{1 z}}{\omega_{1}}\right) S_{j_{1}-1}^{l_{1}-1} \tilde{S}_{j_{2}}^{j_{2}+l_{2}+1}+p_{1 \perp} p_{2 \perp} q_{\perp}\left(1+\frac{\lambda_{1} k_{1 z}}{\omega_{1}}\right)\left(1+\frac{\lambda_{2} k_{2 z}}{\omega_{2}}\right) S_{j_{1}-1}^{l_{1}-1} \tilde{S}_{j_{2}-1}^{j_{2}+l_{2}}\right\}, \\
& v_{22}=-\int \frac{d q_{\perp} q_{\perp}}{\left(q^{2}-M^{2}\right)}\left\{\left[p_{1 z} p_{2 z} q_{z}+p_{1 z}\left(E_{2}+M\right)\left(E_{q}+M\right)+p_{2 z}\left(E_{1}+M\right)\left(E_{q}-M\right)\right.\right. \\
& \left.+q_{z}\left(E_{1}+M\right)\left(E_{2}+M\right)\right] \frac{\lambda_{1} k_{1 \perp} \lambda_{2} k_{2 \perp}}{\omega_{1} \omega_{2}} S_{j_{1}}^{l_{1}} \tilde{S}_{j_{2}}^{j_{2}+l_{2}}+\left[p_{1 z} p_{2 z} q_{z}-p_{1 z}\left(E_{2}+M\right)\left(E_{q}+M\right)\right.
\end{aligned}
$$




$$
\begin{aligned}
& \left.-p_{2 z}\left(E_{1}+M\right)\left(E_{q}-M\right)+q_{z}\left(E_{1}+M\right)\left(E_{2}+M\right)\right]\left(1-\frac{\lambda_{1} k_{1 z}}{\omega_{1}}\right)\left(1+\frac{\lambda_{2} k_{2 z}}{\omega_{2}}\right) S_{j_{1}+1}^{l_{1}} \tilde{S}_{j_{2}-1}^{j_{2}+l_{2}-1} \\
& -\left[p_{1 z} p_{2 z} q_{\perp}+q_{\perp}\left(E_{1}+M\right)\left(E_{2}+M\right)\right] \frac{\lambda_{1} k_{1 \perp}}{\omega_{1}}\left(1+\frac{\lambda_{2} k_{2 z}}{\omega_{2}}\right) S_{j_{1}}^{l_{1}} \tilde{S}_{j_{2}-1}^{j_{2}+l_{2}-1} \\
& -\left[p_{1 \perp} p_{2 z} q_{z}-p_{1 \perp}\left(E_{2}+M\right)\left(E_{q}+M\right)\right] \frac{\lambda_{1} k_{1 \perp}}{\omega_{1}}\left(1+\frac{\lambda_{2} k_{2 z}}{\omega_{2}}\right) S_{j_{1}}^{l_{1}-1} \tilde{S}_{j_{2}-1}^{j_{2}+l_{2}-1} \\
& -\left[p_{1 z} p_{2 \perp} q_{z}+p_{2 \perp}\left(E_{1}+M\right)\left(E_{q}-M\right)\right] \frac{\lambda_{1} k_{1 \perp}}{\omega_{1}}\left(1-\frac{\lambda_{2} k_{2 z}}{\omega_{2}}\right) S_{j_{1}}^{l_{1}} \tilde{S}_{j_{2}+1}^{j_{2}+l_{2}} \\
& -\left[p_{1 \perp} p_{2 z} q_{z}+p_{1 \perp}\left(E_{2}+M\right)\left(E_{q}+M\right)\right] \frac{\lambda_{2} k_{2 \perp}}{\omega_{2}}\left(1+\frac{\lambda_{1} k_{1 z}}{\omega_{1}}\right) S_{j_{1}-1}^{l_{1}-1} \tilde{S}_{j_{2}}^{j_{2}+l_{2}} \\
& +\left[p_{1 z} p_{2 z} q_{\perp}+q_{\perp}\left(E_{1}+M\right)\left(E_{2}+M\right)\right] \frac{\lambda_{2} k_{2 \perp}}{\omega_{2}}\left(1-\frac{\lambda_{1} k_{1 z}}{\omega_{1}}\right) S_{j_{1}+1}^{l_{1}} \tilde{S}_{j_{2}}^{j_{2}+l_{2}} \\
& -\left[p_{1 z} p_{2 \perp} q_{z}-p_{2 \perp}\left(E_{1}+M\right)\left(E_{q}-M\right)\right] \frac{\lambda_{2} k_{2 \perp}}{\omega_{2}}\left(1-\frac{\lambda_{1} k_{1 z}}{\omega_{1}}\right) S_{j_{1}+1}^{l_{1}} \tilde{S}_{j_{2}}^{j_{2}+l_{2}-1} \\
& +p_{1 z} p_{2 \perp} q_{\perp} \frac{\lambda_{1} k_{1 \perp} \lambda_{2} k_{2 \perp}}{\omega_{1} \omega_{2}} S_{j_{1}}^{l_{1}} \tilde{S}_{j_{2}}^{j_{2}+l_{2}-1}-p_{1 \perp} p_{2 z} q_{\perp} \frac{\lambda_{1} k_{1 \perp} \lambda_{2} k_{2 \perp}}{\omega_{1} \omega_{2}} S_{j_{1}}^{l_{1}-1} \tilde{S}_{j_{2}}^{j_{2}+l_{2}} \\
& +p_{1 \perp} p_{2 \perp} q_{z} \frac{\lambda_{1} k_{1 \perp} \lambda_{2} k_{2 \perp}}{\omega_{1} \omega_{2}} S_{j_{1}}^{l_{1}-1} \tilde{S}_{j_{2}}^{j_{2}+l_{2}-1}+p_{1 \perp} p_{2 \perp} q_{\perp} \frac{\lambda_{1} k_{1 \perp}}{\omega_{1}}\left(1-\frac{\lambda_{2} k_{2 z}}{\omega_{2}}\right) S_{j_{1}}^{l_{1}-1} \tilde{S}_{j_{2}+1}^{j_{2}+l_{2}} \\
& -p_{1 \perp} p_{2 \perp} q_{\perp} \frac{\lambda_{2} k_{2 \perp}}{\omega_{2}}\left(1+\frac{\lambda_{1} k_{1 z}}{\omega_{1}}\right) S_{j_{1}-1}^{l_{1}-1} \tilde{S}_{j_{2}}^{j_{2}+l_{2}-1}+p_{1 \perp} p_{2 z} q_{\perp}\left(1+\frac{\lambda_{1} k_{1 z}}{\omega_{1}}\right)\left(1+\frac{\lambda_{2} k_{2 z}}{\omega_{2}}\right) S_{j_{1}-1}^{l_{1}-1} \tilde{S}_{j_{2}-1}^{j_{2}+l_{2}-1} \\
& \left.+p_{1 \perp} p_{2 \perp} q_{z}\left(1+\frac{\lambda_{1} k_{1 z}}{\omega_{1}}\right)\left(1-\frac{\lambda_{2} k_{2 z}}{\omega_{2}}\right) S_{j_{1}-1}^{l_{1}-1} \tilde{S}_{j_{2}+1}^{j_{2}+l_{2}}-p_{1 z} p_{2 \perp} q_{\perp}\left(1-\frac{\lambda_{1} k_{1 z}}{\omega_{1}}\right)\left(1-\frac{\lambda_{2} k_{2 z}}{\omega_{2}}\right) S_{j_{1}+1}^{l_{1}} \tilde{S}_{j_{2}+1}^{j_{2}+l_{2}}\right\} .
\end{aligned}
$$

Here the integral of the triple-Bessel product [32] is given by

$$
\begin{aligned}
S_{n}^{m} & =\int_{0}^{\infty} d r r J_{n}\left(k_{1 \perp} r\right) J_{m-n}\left(q_{\perp} r\right) J_{m}\left(p_{1 \perp} r\right), \\
\tilde{S}_{n}^{m} & =\int_{0}^{\infty} d r r J_{n}\left(k_{2 \perp} r\right) J_{m-n}\left(p_{2 \perp} r\right) J_{m}\left(q_{\perp} r\right) .
\end{aligned}
$$

The $S_{2}$ matrix is derived by exchanging two incident photons in $S_{1}: S_{2}=S_{1}\left(k_{1} \leftrightarrow k_{2}, j_{1} \leftrightarrow j_{2}, \lambda_{1} \leftrightarrow \lambda_{2}\right)$.

[1] M. Uchida and A. Tonomura, Generation of electron beams carrying orbital angular momentum, Nature (London) 464, 737 (2010).

[2] J. Verbeeck, H. Tian, and P. Schattschneider, Production and application of electron vortex beams, Nature (London) 467, 301 (2010).

[3] B. J. McMorran, A. Agrawal, I. M. Anderson, A. A. Herzing, H. J. Lezec, J. J. McClelland, and J. Unguris, Electron vortex beams with high quanta of orbital angular momentum, Science 331, 192 (2011).

[4] K. Saitoh, Y. Hasegawa, K. Hirakawa, N. Tanaka, and M. Uchida, Measuring the Orbital Angular Momentum of Electron Vortex Beams Using a Forked Grating, Phys. Rev. Lett. 111, 074801 (2013).

[5] K. Y. Bliokh, M. R. Dennis, and F. Nori, Relativistic Electron Vortex Beams: Angular Momentum and Spin-Orbit Interaction, Phys. Rev. Lett. 107, 174802 (2011).

[6] S. M. Lloyd, M. Babiker, G. Thirunavukkarasu, and J. Yuan, Electron vortices: Beams with orbital angular momentum, Rev. Mod. Phys. 89, 035004 (2017).
[7] U. D. Jentschura and V. G. Serbo, Generation of High-Energy Photons with Large Orbital Angular Momentum by Compton Backscattering, Phys. Rev. Lett. 106, 013001 (2011); Compton upconversion of twisted photons: Backscattering of particles with non-planar wave functions, Eur. Phys. J. C 71, 1571 (2011).

[8] I. P. Ivanov and V. G. Serbo, Scattering of twisted particles: Extension to wave packets and orbital helicity, Phys. Rev. A 84, 033804 (2011).

[9] L. Allen, M. W. Beijersbergen, R. J. C. Spreeuw, and J. P. Woerdman, Orbital angular momentum of light and the transformation of Laguerre-Gaussian laser modes, Phys. Rev. A 45, 8185 (1992).

[10] Y. Shi, B. F. Shen, L. G. Zhang, X. M. Zhang, W. P. Wang, and Z. Z. Xu, Light Fan Driven by a Relativistic Laser Pulse, Phys. Rev. Lett. 112, 235001 (2014).

[11] I. Bialynicki-Birula and Z. Bialynicka-Birula, Relativistic Electron Wave Packets Carrying Angular Momentum, Phys. Rev. Lett. 118, 114801 (2017). 
[12] S. M. Barnett, Relativistic Electron Vortices, Phys. Rev. Lett. 118, 114802 (2017).

[13] O. G. Rodríguez-Herrera, D. Lara, K. Y. Bliokh, E. A. Ostrovskaya, and C. Dainty, Optical Nanoprobing Via SpinOrbit Interaction of Light, Phys. Rev. Lett. 104, 253601 (2010).

[14] Y. Zhao, J. S. Edgar, G. D. M. Jeffries, D. McGloin, and D. T. Chiu, Spin-to-orbital Angular Momentum Conversion in a Strongly Focused Optical Beam, Phys. Rev. Lett. 99, 073901 (2007).

[15] P. Bandyopadhyay, B. Basu, and D. Chowdhury, Relativistic Electron Vortex Beams in a Laser Field, Phys. Rev. Lett. 115, 194801 (2015).

[16] D. Chowdhury, B. Basu, and P. Bandyopadhyay, Electron vortex beams in a magnetic field and spin filter, Phys. Rev. A 91, 033812 (2015).

[17] P. Bandyopadhyay, B. Basu, and D. Chowdhury, The geometric phase and the geometrodynamics of relativistic electron vortex beams, Proc. R. Soc. London, Ser. A 470, 20130525 (2014).

[18] A. G. Hayrapetyan, O. Matula, A. Aiello, A. Surzhykov, and S. Fritzsche, Interaction of Relativistic Electron-Vortex Beams with Few-Cycle Laser Pulses, Phys. Rev. Lett. 112, 134801 (2014).

[19] D. V. Karlovets, Electron with orbital angular momentum in a strong laser wave, Phys. Rev. A 86, 062102 (2012).

[20] V. Serbo, I. P. Ivanov, S. Fritzsche, D. Seipt, and A. Surzhykov, Scattering of twisted relativistic electrons by atoms, Phys. Rev. A 92, 012705 (2015).

[21] V. P. Kosheleva, V. A. Zaytsev, A. Surzhykov, V. M. Shabaev, and Th. Stöhlker, Elastic scattering of twisted electrons by an atomic target: Going beyond the Born approximation, Phys. Rev. A 98, 022706 (2018).
[22] J. A. Sherwin, Two-photon annihilation of twisted positrons, Phys. Rev. A 98, 042108 (2018).

[23] I. P. Ivanov, N. Korchagin, A. Pimikov, and P. Zhang, Doing Spin Physics with Unpolarized Particles, Phys. Rev. Lett. 124, 192001 (2020).

[24] M. E. Groshev, V. A. Zaytsev, V. A. Yerokhin, and V. M. Shabaev, Bremsstrahlung from twisted electrons in the field of heavy nuclei, Phys. Rev. A 101, 012708 (2020).

[25] M. Katoh, M. Fujimoto, H. Kawaguchi, K. Tsuchiya, K. Ohmi, T. Kaneyasu, Y. Taira, M. Hosaka, A. Mochihashi, and Y. Takashima, Angular Momentum of Twisted Radiation from an Electron in Spiral Motion, Phys. Rev. Lett. 118, 094801 (2017).

[26] J. M. N. Djiokap, S. X. Hu, L. B. Madsen, N. L. Manakov, A. V. Meremianin, and A. F. Starace, Electron Vortices in Photoionization by Circularly Polarized Attosecond Pulses, Phys. Rev. Lett. 115, 113004 (2015).

[27] D. Pengel, S. Kerbstadt, D. Joannmeyer, L. Englert, T. Bayer, and M. Wollenhaupt, Electron Vortices in Femtosecond Multiphoton Ionization, Phys. Rev. Lett. 118, 053003 (2017).

[28] F. C. Vélez, K. Krajewska, and J. Z. Kamiński, Generation of electron vortex states in ionization by intense and short laser pulses, Phys. Rev. A 97, 043421 (2018).

[29] M. W. Beijersbergen, R. P. C. Coerwinker, M. Kristensen, and J. P. Woerdman, Helical-wavefront laser beams produced with a spiral phaseplate, Opt. Commun. 112, 321 (1994).

[30] Y. Y. Liu, Y. I. Salamin, Z. K. Dou, Z. F. Xu, and J. X. Li, Vortex $\gamma$ rays from scattering laser bullets off ultrarelativistic electrons, Opt. Lett. 45, 395 (2020).

[31] A. Longman, C. Salgado, G. Zeraouli, J. Apiñaniz, J. PérezHernández, M. Eltahlawy, L. Volpe, and R. Fedosejevs, Offaxis spiral phase mirrors for generating high-intensity optical vortices, Opt. Lett. 45, 2187 (2020).

[32] A. D. Jackson and L. C. Maximon, Integrals of products of Bessel functions, SIAM J. Math. Anal. 3, 446 (1972). 